\title{
Design and Implementation of Automatic Intelligent Tracking Car Based on CCD
}

\author{
Zhaoran DONG ${ }^{a}$, Mingwei $\mathrm{LI}^{\mathrm{b}}$, Ying XIAO ${ }^{\mathrm{c}}$ \\ College of Information and Communication Engineering,Dalian Nationality University, Dalian, \\ 116600, China \\ aemail: 841536878@qq.com, bemail:1462724048@qq.com, cemail:xiaoying@dlnu.edu.cn
}

\author{
Keywords: Intelligent Tracking car; CCD; Servo Motor; PID Algorithm
}

\begin{abstract}
A method of design and implementation of automatic intelligent tracking car based on CCD was proposed. The automatic intelligent tracking car takes KL26 of Freescale as the controller. The runway information is detected by CCD and the speed of the car is detected by the auxiliary encoder. During the running process of the car, the speed of the car can control by the servo motor with PID algorithm. To achieve real-time running status observation of the automatic intelligent tracking car, PC software is designed. A large number of experimental results show that the automatic intelligent tracking car has stable and reliable performance, which has certain popularization and application value.
\end{abstract}

\section{Introduction}

Automatic intelligent tracking car relates to modern communication technology, automatic control technology and computer technology, which has requirement in a large of engineering applications. The basic principle of automatic intelligent tracking car is that the calculate control unit can implement monitoring and controlling on the running track and driving speed of the car by automatic identification of the route, which can achieve specific goals. The automatic intelligent tracking car does not require human management, which can be competent for some special scientific exploration application, such as high temperature and high pressure environment, toxic and harmful gas environment and outer space exploration environment, etc. where people cannot work directly. Another advantage of automatic intelligent tracking car is low cost, so it is suitable for mass production and wide application, and can be used for survey work without recovering.

There are a variety of design and implementation programs of automatic intelligent tracking car, such as infrared tracking, ultrasonic tracking and cameral tracking, etc. Practice has shown that the automatic intelligent tracking car using CCD to detect the running way has better performance. The automatic intelligent tracking car using CCD return images can not only provide the high precision traveling track information, but also save the store space for small amount of data. Hereby we propose a design and implementation program of automatic intelligent tracking car based on CCD.

\section{System hardware circuit design}

Fig. 1 shows the overall design block diagram of the automatic intelligent tracking car. The controller of the automatic intelligent tracking car uses the KL26 of Freescale, which can detect the speed with the assistant of encoder. By adjusting the speed of the servo motor, the PID algorithm is used to control the speed of the automatic intelligent tracking car. The image information obtained by the automatic intelligent tracking car is uploaded to the host computer through the wireless module, which provides the basic information for the path analysis of the host computer. The system hardware circuit of the automatic intelligent tracking car mainly includes motor driving circuit, CCD camera module and wireless communication module.

The motor driving circuit uses the $\mathrm{N}$ channel MOS tube drive motor. MOS tube is often used in switching power supply, and it is an ideal analog switch device. The 4 MOS tubes are composed of a double $\mathrm{H}$ bridge, when the gate voltage of the 2 MOS tubes reach to $12 \mathrm{~V}$, the MOS tubes are 
turned on and the wheel is turning forward. Otherwise, if the other 2 MOS tubes are turned on, the wheel is turned back. The wheel speed can be changed through the PWM, the duty cycle is large, the speed is fast, and vice versa.

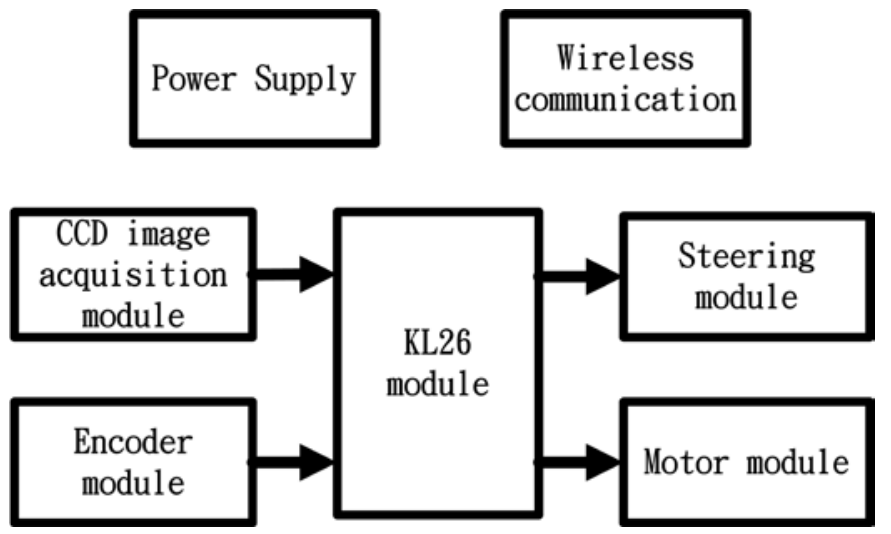

Fig.1 The overall design diagram of the automatic intelligent tracking car

CCD is the key part of the image acquisition, which is responsible for the real-time acquisition of the driving trajectory of the automatic intelligent tracking car. Here we use uses TSL1401 linear $\mathrm{CCD}$, in which $8 \mathrm{AD}$ value is set in program and the gray value range is $0-255$ with the resolution of 128 . Tab. 1 shows the pin functions of CCD.

Tab.1 CCD pin functions

\begin{tabular}{|c|c|c|}
\hline Pin Number & Pin Name & Function \\
\hline 1 & Vout & Ambient brightness value output \\
\hline 2 & SI & Initial control data sequence \\
\hline 3 & GND & Ground \\
\hline 4 & CLK & Pixel output control, frequency $5 \mathrm{k} \sim 8000 \mathrm{khz}$ \\
\hline 5 & VDD & 3V $\sim 5$ DC \\
\hline 6 & AO & Pixel gray value output \\
\hline
\end{tabular}

Wireless communication module realizes the communication and interaction between the running data of the automatic intelligent tracking car and the software system of the host computer. The interface of the host computer is designed by LabVIEW and the travel information is send and received through the Bluetooth module. We can observe and store the 8 channel waveform data which sends by the automatic intelligent tracking car at the host computer. Furthermore, the waveform in the memory can be analyzed by the waveform analysis module.

Lots of testing show that the automatic intelligent tracking car designed based on CCD can stable work and has reliable performance. The travel state of the automatic intelligent tracking car can be clearly monitored through the host computer.

\section{Software Program Design of Single Chip Microcomputer}

The main software program flow chart is shown in Fig.3. The track information is collected by CCD and transferred to digital information after the image processing as de-nosing and two valued etc[1].the deviation of the automatic intelligent tracking car can be calculated real-time, according to which to control the servo motor spin acceleration or deceleration.

The principle of CCD is to use high-sensitivity semiconductor materials to convert light into electric charge. The analog signal is converted into digital signal through digital analog conversion and transmitted to the single chip microcomputer. And then a series of algorithms is carried on to deal with the situation to determine the runway. The advantage of CCD is far away from the outside and has high resolution under the high light interference, which equals to 128 photoelectric tubes[2]. Furthermore, the program is relatively simple and easy to control. The image acquisition and processing process is shown in Fig.4. 


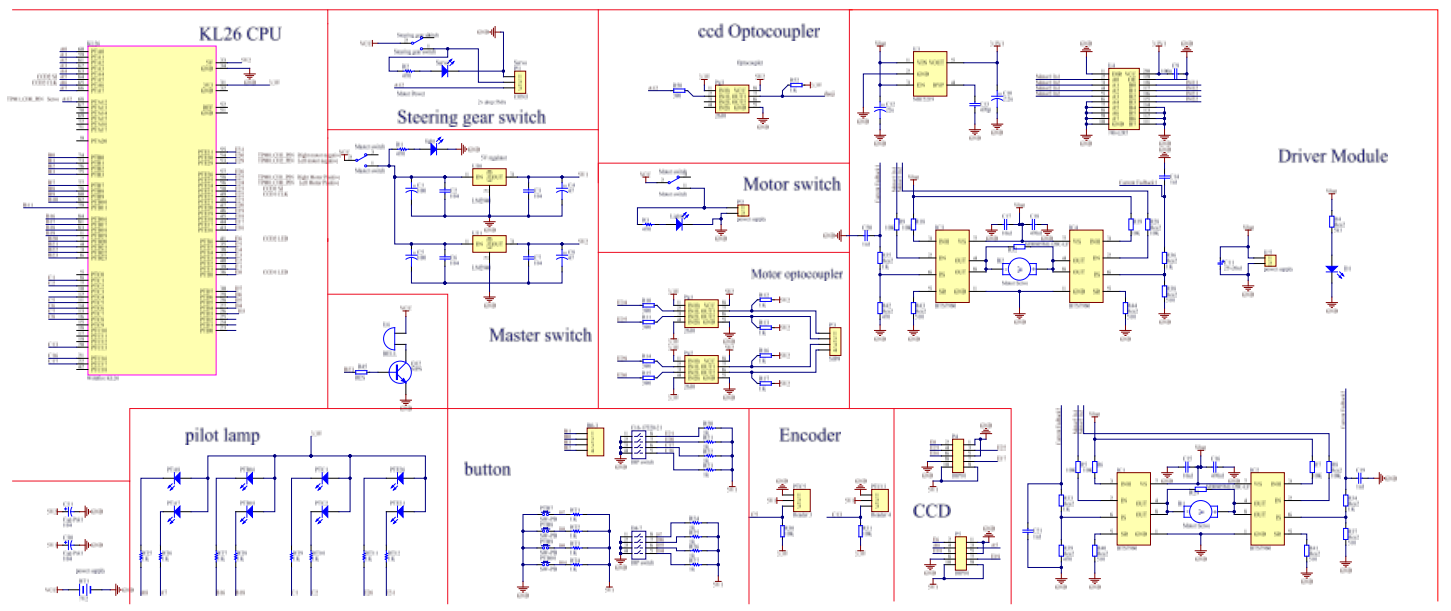

Fig.2 Circuit schematics of the communication module

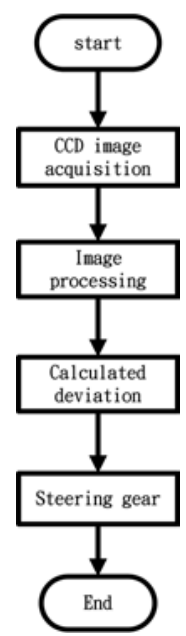

Fig.3 The main software program flow chart

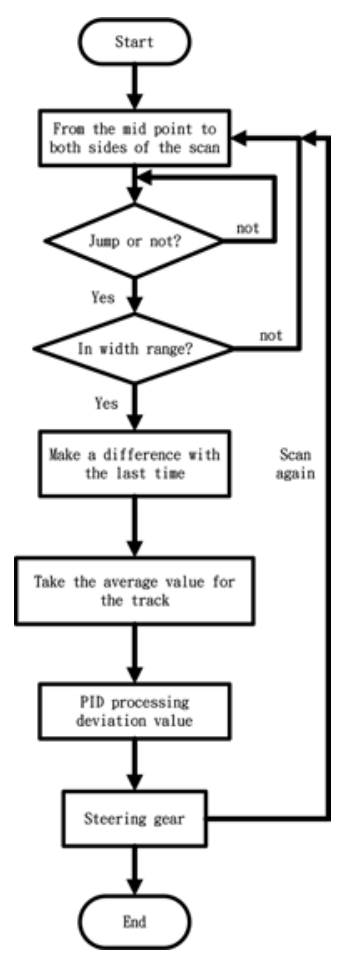

Fig.4 The image acquisition and processing process

During the running process, the speed of the automatic intelligent tracking car needs real-time adjusting by using the running way information, especially for the slope road and curving road, the automatic intelligent tracking car must control its speed at a safe range[3]. The speed of the 
automatic intelligent tracking car can be controlled by the servo motor, whose speed is controlled by the PID controller. The PID controller is a kind of parallel negative feedback controller combined with the mathematical theory of proportion, integral and differential, which can calculate the deviation according to the expected output and the real output. The control equation is given by

$$
U(t)=K_{p}\left[e(t)+\frac{1}{T_{i}} \int_{0}^{t} e(t) d t+\frac{T_{D} d e(t)}{d t}\right]
$$

where $K_{p}$ is the ratio coefficient, $T_{i}$ is the integral time constant and $T_{D}$ is the differential time constant.[4]

The control motor speed is controlled by incremental PID algorithm, which can implement straight acceleration and curve deceleration. The automatic intelligent tracking car needs to decelerate before it enter the curing road, and keep a fixed speed to pass the curing road. When the automatic intelligent tracking car leaves the curing road, it must accelerate in advance. Especially over $\mathrm{S}$ bending and 90 degrees right angle bending road, the car needs to be treated separately. After several experiments found that the speed is set to two times the curve effect is better, the car stability is higher[5]. The PID control system is shown in Fig.5.

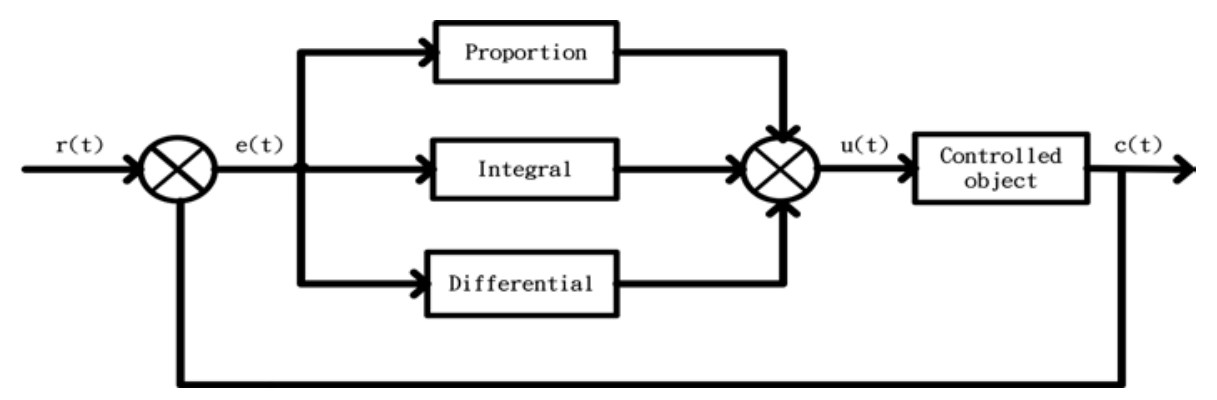

Fig. 5 PID control system schematics

\section{Test and Analysis}

The automatic intelligent tracking car is composed of three parts, which are car model chassis, front wheel servo steering engine and rear wheel drive motor. The size of the car model chassis is $26 \times 17.3 \times 29.5 \mathrm{~cm}$, diameter of front wheel is $32.2 \mathrm{~mm}$ and there filled with sponge to reach the purpose of damping. Servo steering gear of front wheel uses S3010 steering gear, which has low cost and high torque. Under the normal condition, the steering engine operating current is $130+/-25 \mathrm{~mA}$. The stall operating current is $15 \mathrm{~mA}$ with size is $40 \times 20 \times 38.1 \mathrm{~mm}$. When the voltage is $6 \mathrm{~V}$, the torque is $6.5 \pm 1.3 \mathrm{~kg} / \mathrm{cm}$ and the speed is $0.16+0.02 \mathrm{sec} / 60$ with the angle is $60 \pm 10$ degree. The speed of the real wheel can be controlled by PWM signal of the car. The overall layout of the automatic intelligent tracking car designed based on CCD is shown in Fig.6.

The Bluetooth module and the host computer software are used to test the automatic intelligent tracking car, including the collected image, debug deviation and threshold value etc[6]. After a large number of different path testing, we have analyzed and recognized the running information of the automatic intelligent tracking car. The results show that the automatic intelligent tracking car designed based on CCD has good stable performance and the data collecting and the speed controlling is sensitive and reliable. 


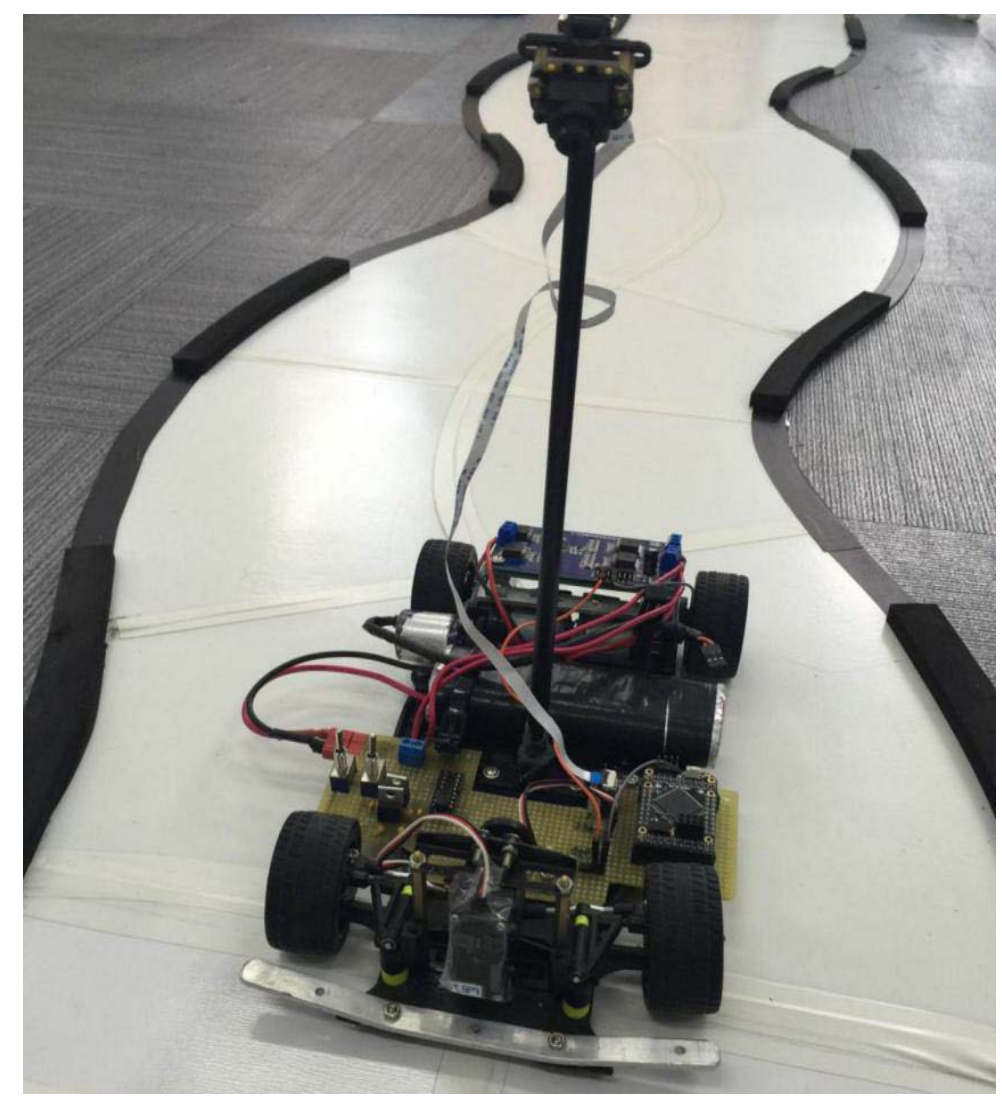

Fig.6 The overall layout of the automatic intelligent tracking car

\section{Conclusion}

This work introduces a design and implementation of automatic intelligent tracking car based on CCD, in which the CCD is adopted as the running information collecting device. The motor speed is controlled by PID algorithm and the human computer interaction is through the host computer software system, which can achieve the real-time monitoring and analysis of the running parameters of the automatic intelligent tracking car. A large number of test results prove that the automatic intelligent tracking car is stable and reliable in all kinds of road conditions, and has certain application value in practical engineering.

\section{References}

[1] Zili Zou . Colorimetric Measurement System and Its Algorithm Based on Linear Array CCD . Applied Physics, 2013, Vol.03 (07), pp.141-148

[2] Minjie Yuan . Design of Driving Linear CCD in Laser Displacement Sensor . Open Journal of Circuits and Systems, 2015, Vol.04 (02), pp.23-31

[3] YANG Ming . Research on Methods of Smartcar' s Servo Control Based on Photodiode Route Recognition .ELECTRO-OPTIC TECHNOLOGY APPLICATION,Vol.22, No.1 February.2007

[4] WU Qing . Multi-Channel Control Signal of Electrical Steering Gear Based on Time-Division Multiplexing PWM Module .

http://www.cnki.net/kcms/detail/31.1428.TM.20160823.1522.058.htm,2016-08-23 15:22:54

[5] LIU Shihong . Study of intelligent vehicle servo control system based on PID neural network . Industrial Instrumentation \& Automation | Ind Instrumm Autom . 2014.

[6] Wang Ling . The research and design of intelligent autonomous tracing car measurement and control system . Engineering Sciences ,2014. 